Tropical Journal of Pharmaceutical Research September 2019; 18 (9): 1919-1925

ISSN: 1596-5996 (print); 1596-9827 (electronic)

(1) Pharmacotherapy Group, Faculty of Pharmacy, University of Benin, Benin City, 300001 Nigeria.

\title{
Effect of hyaluronic acid: Mechanistic investigations via topological and functional analysis of its protein interaction network
}

\author{
Ji Xi ${ }^{1,2}$, Yu Li Ting ${ }^{1}$, Li Xue ${ }^{1}$, Luo Dan ${ }^{2 *}$ \\ ${ }^{1}$ Department of Dermatology, Women's Hospital of Nanjing Medical University, Nanjing Maternity and Child Health Care \\ Hospital, ${ }^{2}$ Department of Dermatology, The First Affiliated Hospital of Nanjing Medical University, Nanjing 210029, China \\ ${ }^{*}$ For correspondence: Email: dannyluo2019@sina.com; Tel: 0086-13951808478 \\ Sent for review: 26 April 2019 \\ Revised accepted: 26 August 2019
}

\begin{abstract}
Purpose: To predict molecular targets and the mechanism of action of hyaluronic acid against various disease conditions.

Methods: The present study was designed and conducted based on the principle of network pharmacology, protein-protein interaction (PPI) database, and biological process analysis. At a high confidence score, a protein network comprising seventeen human protein targets was achieved from STITCH (Search Tool for Interaction of Chemicals).

Results: Cytoscape-based protein-protein interaction network (PPIN) with 342 nodes was developed and analyzed to ascertain the molecular basis of hyaluronic acid actions. It revealed that hyaluronic acid was mainly involved in various physiological processes including extracellular matrix (ECM) disassembly, collagen catabolic process, mucopolysaccharide metabolic process, and circadian regulation of gene expression.

Conclusion: The retrieved pathways are known to be involved in causing several pathologies such as leukemia, psoriasis, tick-borne encephalitis, etc. These pathways are influenced by hyaluronic acid. However, further explanations are required to elucidate whether these targets of hyaluronic acid have a therapeutic or toxicological role.
\end{abstract}

Keywords: Hyaluronic acid, Network pharmacology, Molecular targets, STITCH, Cytoscape, Biological effects

\begin{abstract}
This is an Open Access article that uses a fund-ing model which does not charge readers or their institutions for access and distributed under the terms of the Creative Commons Attribution License (http://creativecommons.org/licenses/by/4.0) and the Budapest Open Access Initiative (http://www.budapestopenaccessinitiative.org/read), which permit unrestricted use, distribution, and reproduction in any medium, provided the original work is properly credited.

Tropical Journal of Pharmaceutical Research is indexed by Science Citation Index (SciSearch), Scopus, International Pharmaceutical Abstract, Chemical Abstracts, Embase, Index Copernicus, EBSCO, African Index Medicus, JournalSeek, Journal Citation Reports/Science Edition, Directory of Open Access Journals (DOAJ), African Journal Online, Bioline International, Open-J-Gate and Pharmacy Abstracts
\end{abstract}

\section{INTRODUCTION}

Hyaluronic acid is a non-sulfated glycosaminoglycan [1]. In humans, hyaluronic acid mainly exists (50\% of total body hyaluronic acid contents) in the skin [2]. One of the main features of hyaluronic acid is its capability to hold water molecules, thus it plays an important role in maintaining skin moisture and health. The reduced contents of hyaluronic acid in the skin leads to aging. Thus, it is potentially used in cosmeceutical preparations for anti-aging effect [3]. However, the molecular targets and the associated mechanisms of hyaluronic acid are still imprecisely known. Therefore, studies are required to identify the molecular targets of 
hyaluronic acid effects and the pertinent therapeutic modes of hyaluronic acid.

Network pharmacology is being increasingly utilized to assess the constituents and mechanism of action of herbs. Network pharmacology is a system biology-based approach that has helped the medicine scientists accelerate the process of drug discovery by investigating the scientific validation of drug action $[4,5]$.

The performance of physiological functions depends on various protein-protein interaction (PPI) networks that comprise a large number of proteins connected through cellular pathways and perform the biological functions [5]. On the basis of gene ontology (GO) project, gene ontology annotations have been created which are useful in performing enrichment analysis on gene sets [6].

The objective of this study was to investigate in silico the molecular basis of hyaluronic acid effects using network pharmacology. This study not only acts as a reference for clinical trials of hyaluronic acid but can also be helpful to fabricate its analogs. In the accomplishment of this study, Cytoscape was used for constructing protein-protein interaction networks (PPINs) which were subsequently analyzed using GO enrichment modality to recognize and analyze the useful modules.

\section{METHODS}

\section{Protein targets retrieval}

STITCH 5.0 database (http://stitch.embl.de/) [7] was utilized to retrieve protein targets of hyaluronic acid and develop a PPI network. This online database provides information from multiple sources including experimental validations and text mining to scrutinize protein targets of an active compound and their interacting networks. STITCH database provides interaction potential of a compound in terms of a probabilistic confidence score [8]. The targets having a high confidence score $(>0.7)$ with not more than 20 interactors were obtained to develop a PPI network.

\section{Protein interaction network}

Cytoscape (version 2.8.2; http://www.cytoscape. org/) was used to obtain and analyze PPIN. Cytoscape is a Java ${ }^{\circledR}$-based software that is supplied with a wide range of plug-ins [9]. This software was used to merge the individual networks via graph union operation for the construction of large complex PPIN. This operation helps to merge duplicated edges and self-loops retrieved through reciprocal interaction finding and graph merging approaches to get rid of duplication if any. Moreover, undirected treatment of network, i.e. a treatment supposing similar nature of the vertices, was opted for this study.

\section{Topological analysis of network}

Topological analysis is a useful tool for understanding the protein interaction network [10]. Therefore, different topological parameters such as clustering coefficient, the number of nodes, connected components, network density, network diameter, isolated nodes, network radius, the average number of neighbors, network centralization, number of self-loops, network heterogeneity and characteristic path length were determined using Network Analyzer in Cytoscape [11].

\section{Analysis of biological processes}

ClueGO, a plug-in of Cytoscape [12], was utilized for biological process analysis to investigate biological characteristics of target genes associated with hyaluronic acid. Cytoscape is a useful tool for visualizing gene functionality in the form of a cluster as well as a statistical chart. Two-sided hypergeometric test with a Bonferroni correction and the level of significance set at 0.05 for a medium type network was used for the enrichment analysis with an organic layout algorithm for functional network visualization.

\section{RESULTS}

\section{Retrieval of protein targets}

Using STITCH (accessed in April 2017) at a high confidence score (0.7), 17 different potential human protein targets of hyaluronic acid (Table 1, Figure 1) were retrieved. These protein targets play the important roles in various physiological and/or pathological processes. In order to simplify the description of the pharmacological significance of these 17 targets of hyaluronic acid, the 17 protein targets were further analyzed through Cytoscape plugin ClueGO. As a result, three important GO terms i.e. the extracellular matrix (ECM) disassembly, collagen catabolic process, and mucopolysaccharide metabolic process (having GO ID 22617, 30574 and 1903510, respectively) were found. Their associated genes found in this analysis were ACAN, ADAMTS4, KLK4, MMP13 and MMP9 for 22617, ADAMTS3, MMP13 and MMP9 for 30574 
and ACAN, HAS2, HMMR, HYAL2, IL6, SPAM1, STAB2 and VCAN for 1903510, respectively. The findings of this study reveal that hyaluronic acid could be involved in the breakdown of ECM especially collagen and related materials, showing its potential role as a regenerative medicine.

Table 1: Predicted targets of hyaluronic acid (Confidence view)

\begin{tabular}{ll}
\hline Protein target & Score \\
\hline CD44 & 0.981 \\
ADAMTS4 & 0.906 \\
HAS2 & 0.900 \\
IL6 & 0.873 \\
HYAL2 & 0.872 \\
MMP13 & 0.872 \\
MMP9 & 0.871 \\
ADAMTS3 & 0.860 \\
VCAM1 & 0.828 \\
SPAM1 & 0.826 \\
HMMR & 0.808 \\
KLK4 & 0.800 \\
TNFSF11 & 0.800 \\
VCAN & 0.741 \\
ACAN & 0.739 \\
STAB2 & 0.723 \\
HAPLN1 & 0.715 \\
\hline
\end{tabular}

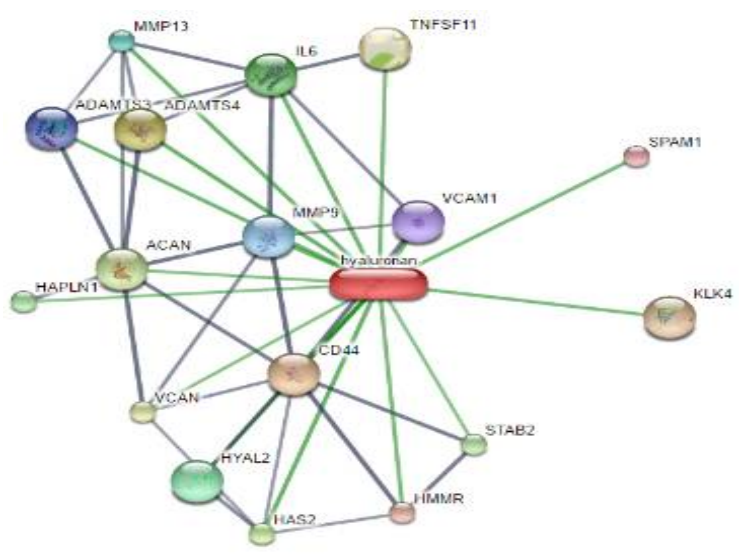

Figure 1: Hyaluronic acid protein network (confidence view) showing nodes and edges that denote proteins and their interactions, respectively. Thick lines denote stronger linkages. Grey and green lines symbolize the protein-protein and chemical-protein linkages, respectively. (ADAMTS4 - ADAM metallopeptidase with thrombospondin type 1 motif, 4: HAS2 hyaluronan synthase 2; IL6 - interleukin 6: HYAL2 hyaluronoglucosaminidase 2; MMP13 - matrix metallopeptidase 13; MMP9 - matrix metallopeptidase 9: ADAMTS3 - ADAM metallopeptidase with thrombospondin type 1 motif, 3: VCAM1 - vascular cell adhesion molecule 1; SPAM1 - sperm adhesion molecule 1; HMMR - hyaluronan-mediated motility receptor (RHAMM); KLK4 - kallikrein-related peptidase 4; TNFSF11 - tumor necrosis factor (ligand) superfamily, member 11; VCAN - versican; ACAN aggrecan; STAB2 - stabilin 2; HAPLN1 - hyaluronan and proteoglycan link protein 1)

\section{Protein interaction network}

The protein interaction network was imported in Cytoscape and union calculations were made. Subsequently, the networks were merged using the advance network merge plug-in to ensure the removal of the duplicated edges. As a result, PPIN of hyaluronic acid (Figure 2) comprising 342 nodes was accomplished, where nodes and edges represented proteins and their interactions, respectively.

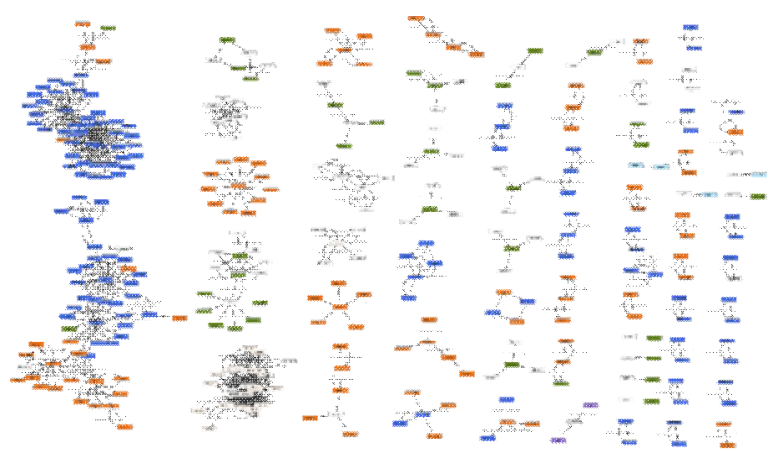

Figure 2: Protein interaction network of hyaluronic acid

\section{Topological analysis of network}

From the topological analysis, 69 connected components of PPIN were observed. Network heterogeneity value was 1.380 showing the extent of uneven distribution of the network. Network diameter was 8 that represented the maximum distance between any pair of vertices. Network centralization value was 0.073: it showed the degree of dispersion of all node centrality scores in a network. The remaining parameters are detailed in Table 2.

Table 2: Topological features of hyaluronic acidprotein interaction network as assessed by Cytoscape Network Analyzer

\begin{tabular}{lc}
\hline Topological parameter & Value \\
\hline Clustering coefficient & 0.01 \\
Connected components & 69 \\
Network diameter & 8 \\
Network radius & 1 \\
Network centralization & 0.07 \\
Network heterogeneity & 1.38 \\
Number of nodes & 342 \\
Network density & 0.01 \\
Isolated nodes & 3 \\
Mean number of neighbors & 2.22 \\
Number of self-loops & 4 \\
Characteristic path length & 2.88 \\
\hline
\end{tabular}




\section{Analysis of biological processes}

Due to versatile biochemical features and specific interaction with cells and ECM constituents in the body, hyaluronic acid has been successfully utilized in various biomedical fields such anti-adhesion, ophthalmology, dermatology and wound healing, chondroprotective and orthopedic applications. However, network pharmacology approach utilizing biological processes analysis revealed some other bioactivities as detailed below, which are not documented earlier.

In functional enrichment analysis, the biological functions of hyaluronic acid-related targets were annotated using gene ontology terms (GO terms). Subsequently, biological process terms (BP terms) were evaluated using Cytoscape plugin ClueGO. These two evaluations showed a significant enrichment of $103 \mathrm{GO}$ terms, which were categorized into 11 groups (Figure 3). These groups were largely involved in circadian regulation of various gene expression, such as protein trimerization, response to retinoic acid, retinoic acid receptor (RAR) signaling pathway, and positive regulation of autophagy.

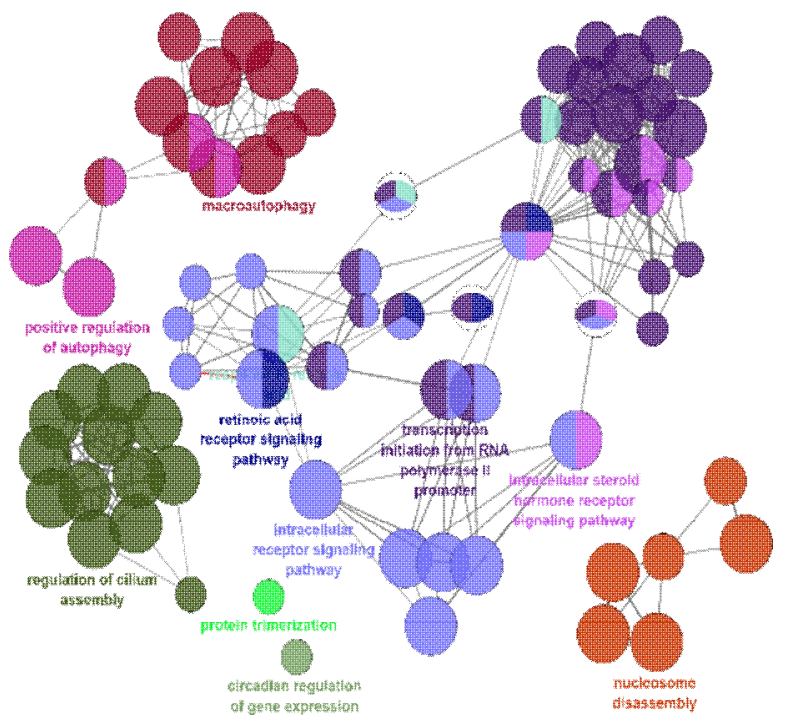

Figure 3: Functionally grouped networks, determined through ClueGO analysis to retrieve the potential targets of hyaluronic acid. Each cluster consists of the most crucial terms only. The overlapped clusters reflect a resemblance of their functions

\section{DISCUSSION}

Biologically, ECM refers to extracellular molecules (for instance, minerals, fibrous proteins, proteoglycans, and glycosaminoglycans) released from the cells, which furnish the surrounding cells with structural and biochemical support for the hematopoietic elements [13]. ECM disassembly leads to the collapse of the extracellular matrix by proteases, for example, matrix metalloproteinases. Thus, ECM disassembly is an important step, not only in normal physiological functions including tissue remodeling but also in pathological states including cancer development [14]. Hyaluronic acid has emerged as a primitive polymer having excellent viscoelastic, hygroscopic, and scaffold characteristics of ECM component. Moreover, hyaluronic acid interacts with cell membrane receptors such as CD44 leading to various signaling pathways. This interaction results in the proliferation, migration, and differentiation of cells, especially in the epidermal layer of the skin due to its short half-life in the skin. Here, hyaluronic acid shows its active metabolic property, particularly in chemical reactions and pathways involving glycosaminoglycans. These polysaccharides lubricate joints and produce cartilage matrix [15-17].

Circadian regulation of gene expression, also termed as diurnal variation of gene expression, deals with the extent of gene expression as a function of time or environmental factors [17]. Circadian regulation is linked with a wide range of biological processes including detoxification, cognition, locomotion, metabolism, and vision [18]. Thus, these activities could be modulated by hyaluronic acid, as found in this study. This study also reports that hyaluronic acid affects protein trimerization, which involves the synthesis of a stable macromolecular structure of a protein trimer. A trimer contains three noncovalently linked similar or dissimilar subunits. Protein trimerization is associated with various diseases such as psoriasis, tick-borne encephalitis, atherosclerosis, stomatitis, and autoimmune diseases, and it also plays a crucial role in the synthesis of vaccines and various therapeutic agents [19].

The findings of the present study describe that the response to retinoic acid and RAR signaling pathways could also be mediated by hyaluronic acid. The response to the retinoic acid pathway is marked by different genes such as KMT2E, NCOA1, RARA, RARG, RXRA, and TRIM16. In this biological process, retinoic acid stimulus induces some modifications in cellular activity in terms of gene expression and enzyme production. Many cancers such as leukemia and embryonal carcinoma have been correlated with a response to retinoic acid [20], and the same result has been observed in the present study. There are several studies that have documented the association of retinoic acid responsiveness with the differentiation, proliferation, growth, and death of the cell. Moreover, RAR signaling 
pathway is a bioprocess in which the binding of retinoic acid with one of its physiological ligands leads to the generation of a series of molecular signals.

Retinoic acid receptor signaling plays an important role during development, differentiation, proliferation and apoptosis of cells. Retinoic acid produced in the particular sites is involved in the regulation of transcription by interacting with nuclear retinoic acid receptors associated with retinoic acid response elements near target genes [21]. Retinoic acid is vital for developing and functioning of multiple organ system. However, deregulation of retinoic acid signaling can contribute to the lethal diseases. Many retinoic acid derivatives or analogs are in the process of clinical trials against various diseases including cancer [22]. This fact signifies the importance of the present study, since it proposes the role of hyaluronic acid in the RAR signaling pathway.

Various studies have described the upregulation of autophagy in response to intra- or extracellular stress and signaling pathways which are involved in microbial infection and growth factor deprivation. The defects in autophagy are responsible for various diseases such as infections, cancer, and neurodegenerative disorders [8]. The present study has revealed the involvement of hyaluronic acid in the positive regulation of autophagy that is associated with more than 65 genes. Out of these 65 genes, 53 are linked with positive regulation of macroautophagy genes. Hyaluronic acid is predicted to positively regulate macroautophagy through several genes such as ATG3, ATG4B, ATG7, BNIP3L, FAM134B, GABARAP, GABARAPL1, GABARAPL2, OPTN, SQSTM1, STBD1, TBC1D25, TP53INP1, TP53INP2, and ULK1. Macroautophagy is a process that is characterized by lysosomal degradation and recycling of cellular components [23].

Transcription initiation from RNA polymerase II promoter could also be affected by hyaluronic acid as evident from Figure 3. During this biological process, the synthesis of RNA polymerase II pre-initiation complex at an RNA polymerase II promoter region of a DNA template takes place [13]. Thus, the synthesis of a number of transcription factors could be affected by hyaluronic acid. Moreover, hyaluronic acid could also induce the generation of intracellular receptor signals due to binding of hormones especially steroid hormones such as estrogen, androgen, and progesterone that lead to changes in gene expression [24].
Hyaluronic acid regulates cilium assembly which is affected by phosphorylation of multiple protein kinases including mitogen-activated protein kinases, aurora kinases, and cyclin-dependent protein kinases [19]. Cilia, especially motile cilia, are microtubules present on the cell surface and are involved in governing functions of cells such as signaling. Many studies have reported the interplay between cilia and autophagy, i.e. autophagosome formation is triggered by cilia. On the other hand, ciliogenesis is regulated by autophagy [25]. This interplay reveals the association of cell biology with human pathologies [25]. Thus, hyaluronic acid could be useful in treating chronic diseases such as cancer due to its possible involvement in autophagy.

Hyaluronic acid also mediates nucleosome disassembly, i.e. the controlled breakdown of nucleosomes and is linked with DNA replication, histone acetylation and cell cycle [8]. This biological process is associated with leukemia [12].

At present, no study is available that document reliable scientific confirmations to correlate these genes with system effects of hyaluronic acid including circadian regulation of gene expression, protein trimerization, response to retinoic acid, and retinoic acid receptor (RAR) signaling pathway. However, this study provides a comprehensive picture of modes of action of hyaluronic acid in these biological processes, which could be experimentally clarified in the future.

\section{CONCLUSION}

In the course of finding therapeutic mechanisms, protein interaction network analysis disclosed the participation of hyaluronic acid in different pathways including extracellular matrix (ECM) disassembly, collagen catabolic process, mucopolysaccharide metabolic process, and circadian regulation of gene expression. These pathways have an association with several pathologies Since it is not clear from the results whether these targets of hyaluronic acid possess therapeutic role or not, these findings should be scientifically validated in future investigations including docking studies. This in silico study proposes an exciting way to recognize the mechanism of action of hyaluronic acid in different pathologic conditions. Likewise, clinical investigations of hyaluronic acid and scheming of its suitable analogs can be supported by this study. 


\section{DECLARATIONS}

\section{Conflict of interest}

No conflict of interest is associated with this work.

\section{Contribution of authors}

We declare that this work was done by the authors named in this article and all liabilities pertaining to claims relating to the content of this article will be borne by the authors.

\section{Open Access}

This is an Open Access article that uses a funding model which does not charge readers or their institutions for access and distributed under the terms of the Creative Commons Attribution License (http://creativecommons.org/licenses/by/ 4.0) and the Budapest Open Access Initiative (http://www.budapestopenaccessinitiative.org/rea d), which permit unrestricted use, distribution, and reproduction in any medium, provided the original work is properly credited.

\section{REFERENCES}

1. Nobile V, Buonocore D, Michelotti A, Marzatico F. Antiaging and filling efficacy of six types hyaluronic acidbased dermo-cosmetic treatment: double blind, randomized clinical trial of efficacy and safety. J Cosmetic Dermatol 2014; 13: 277-287.

2. Papakonstantinou E, Roth M, Karakiulakis G. Hyaluronic acid: a key molecule in skin aging. Dermatoendocrinol 2012; 4: 253-258.

3. Mustoe TA, Gurjala A. The role of the epidermis and the mechanism of action of occlusive dressings in scarring. Wound Repair Regen 2011; 19(Suppl 1): s16-21.

4. Li S, Zhang B. Traditional Chinese medicine network pharmacology: theory, methodology and application. Chinese J Nat Med 2013; 11: 110-120.

5. Cheng D, Murtaza G, Ma S, Li L, Li X, Tian F, Zheng J, Lu Y. In Silico Prediction of Anti-Depression Mechanism of a Herbal Formula (Tiansi Liquid) containing Morinda officinalis and Cuscuta chinensis. Molecules 2017; 22(10): 1614-1630.

6. Janga SC, Tzakos A. Structure and organization of drugtarget networks: insights from genomic approaches for drug discovery. Mol Biosyst 2009; 5: 1536-1548.

7. Yao X, Hao H, Li Y, Li S. Modularity-based credible prediction of disease genes and detection of disease subtypes on the phenotype-gene heterogeneous network. BMC Syst Biol 2011; 5: 79-85.

8. Kuhn M, Szklarczyk D, Pletscher-Frankild S, Blicher TH, von Mering $C$, Jensen LJ. STITCH 4: integration of protein-chemical interactions with user data. Nucleic Acids Res 2014; 42: 401-407

9. Smoot ME, Ono K, Ruscheinski J, Wang PL, ldeker T. Cytoscape 2.8: new features for data integration and network visualization. Bioinform 2011; 27: 431-432.

10. AlbertR. Scale-free networks in cell biology. J Cell Sci 2005; 118: 4947-4957.

11. Gaulton A, Bellis LJ, Bento AP, Chambers J, Davies M, Hersey A. ChEMBL: a large-scale bioactivity database for drug discovery. Nucleic Acids Res 2012; 40: D11001107.

12. Bindea G. ClueGO: a Cytoscape plug-in to decipher functionally grouped gene ontology and pathway annotation networks. Bioinformatic 2009; 25: 10911093.

13. Benders KE, van Weeren PR, Badylak SF, Saris DB, Dhert WJ, Malda J. Extracellular matrix scaffolds for cartilage and bone regeneration. Trends Biotechnol 2013; 31(3): 169-176.

14. Lu P, Takai K, Weaver VM, Werb Z. Extracellular Matrix Degradation and Remodeling in Development and Disease. Cold Spring Harb Perspect Biol 2011; 3(12): a005058.

15. Wellen KE, Thompson CB. Cellular metabolic stress: considering how cells respond to nutrient excess. Mol Cell 2010; 40(2): 323-332.

16. Assenov $Y$, Ramirez $F$, Schelhorn SE, Lengauer $T$, Albrecht M. Computing topological parameters of biological networks. Bioinform 2008; 24: 282-284.

17. Manke $T$, Demetrius L, Vingron M. Lethality and entropy of protein interaction networks. Genome Inform 2005; 16: 159-163.

18. Claridge-Chang A, Wijnen $H$, Naef $F$, Boothroyd $C$, Rajewsky N, Young MW. Circadian regulation of gene expression systems in the Drosophila head. Neuron 2001; 32: 657-671.

19. Sliepen K, van Montfort T, Melchers M, Isik G, Sanders $R W$. Immunosilencing a highly immunogenic protein trimerization domain. J Biochem 2015, doi: 10.1074/jbc.M114.620534.

20. Kasimanickam VR, Kasimanickam RK, Dernell WS. Dysregulated microRNA Clusters in Response to Retinoic Acid and CYP26B1 Inhibitor Induced Testicular Function in Dogs. PLoS One 2014; 9(6): e99433. https://doi.org/10.1371/journal.pone.0099433

21. Cunningham TJ, Duester G. Mechanisms of retinoic acid signalling and its roles in organ and limb development. Nature Reviews Molecul Cell Biol 2015, 16: 110-123.

22. Das BC, Thapa P, Karki R, Das S, Mahapatra S, Liu TC, Torregroza I, Wallace DP, Kambhampati S, Veldhuizen $P V$, et al. Retinoic Acid Signaling Pathways in Development and Diseases. Bioorg Med Chem 2014; 22(2): 673-683.

23. Feng $Y$, He $D$, Yao Z, Klionsky DJ. The machinery of macroautophagy. Cell Res 2014; 24: 24-41.

24. Dahlman-Wright $K$, Cavailles V, Fuqua SA, Jordan VC, Katzenellenbogen JA, Korach KS, Maggi A, Muramatsu $M$, Parker MG, Gustafsson JA. International Union of

Trop J Pharm Res, September 2019; 18(9): 1924 
Pharmacology. LXIV. Estrogen receptors. Pharmacol Rev 2006; 58 (4): 773-781.
25. Orhon I, Dupont N, Pampliega O, Cuervo AM, Codogno $P$. Autophagy and regulation of cilia function and assembly. Cell Death Differ 2015; 22: 389-397. 